\title{
TARGET SCORE OF RIQAS AND SIGMA METRICS FOR EVALUATING THE ANALYTICAL PERFORMANCE OF THYROID FUNCTION TESTING ON ADVIA CENTAUR XPT IMMUNOASSAY ANALYSER
}

\author{
Babu Rao Rentapalli1, Suresh Babu Ganji², M. D. Dawood Sulemani ${ }^{3}$ \\ ${ }^{1}$ Associate Professor, Department of Biochemistry, GMC, Hyderabad, Telangana, India. \\ ${ }^{2}$ Associate Professor, Department of Biochemistry, MNJIO, OMC, Hyderabad, Telangana, India. \\ 3 Professor and HOD, Department of Biochemistry, Gandhi Medical College and Hospital, Hyderabad, Telangana, India.
}

ABSTRACT

\section{BACKGROUND}

Quality test reporting is essential part of diagnosis in any aliment, especially where the symptoms and signs are subtle like in thyroid disease. Quality assurance in clinical laboratory is presently done by both internal and external quality programs. RIQAS program Target Score is a performance indicator and sigma metrics is considered as a gold standard for overall performance.

The ai of this study was to present the Target Scoring (TS) and Sigma Metrics observed in our clinical chemistry laboratory in a tertiary care hospital to evaluate the analytical performance of T3, T4, TSH parameters.

\section{MATERIALS \& METHODS}

The thyroid function parameters Bio-Rad daily controls run on Siemens ADVIA Centaur XPT Immunoassay analyser to monitor the CV; and returned results of Target Score, SDI and \%age Deviation of monthly run controls of Randox RIQAS external quality program are taken for a consecutive three months period for this study. Sigma values calculated and quality control interpreted.

\section{RESULTS}

Our EQAS results showed two thirds of good performances. In August, the 3 tests were performing well. But in the month of September, TSH was having a very low TS; and SDI, \% Deviation were outside limits. In October, T3 results were not acceptable and TSH analysis needed improvement. Only T4 results were shown to be consistent and steady. On the other side, sigma metrics were below three sigmas for all the 3 months as we had a wider imprecision (CV).

\section{CONCLUSION}

Quality check strategies were applied according to the TS and sigma values.

\section{KEY WORDS}

RIQAS: Randox International Quality Assurance Scheme, TS: Target Score, Sigma Metrics, Thyroid Function Test, QC: Quality Control.

HOW TO CITE THIS ARTICLE: Rentapalli BR, Ganji SB, Sulemani MDD. Target score of riqas and sigma metrics for evaluating the analytical performance of thyroid function testing on advia centaur XPT immunoassay analyser. J. Evolution Med. Dent. Sci 2019;8(07):447-450, DOI: $10.14260 /$ jemds/2019/98

\section{BACKGROUND}

Thyroid dysfunction is prevalent in clinical practice and has significant consequences. Quality laboratory tests are essential for the accurate diagnosis of thyroid disorders because the signs and symptoms of thyroid disease are subtle or absent in most patients, making biochemical tests necessary to detect disease. ${ }^{1}$ The Target Score (TS) allows you to assess your performance at a glance. Target Score is a performance indicator for RIQAS only, unique tool of RIQAS. ${ }^{2}$ Six sigma is a technique to quantify errors or defects, i.e., nonconformances (NC) like false positives, false negatives and outliers in the laboratory. A Six Sigma analytical method is one that is expected to generate less than four erroneous results per million test reports.

\section{'Financial or Other Competing Interest': None.}

Submission 28-12-2018, Peer Review 01-02-2019,

Acceptance 09-02-2019, Published 18-02-2019.

Corresponding Author:

Suresh Babu Ganji,

F-211-B Block,

Sumadhura Elegance Apartment,

SBH-C Colony, Saidabad,

Hyderabad-500059,

Telangana, India.

E-mail: sushwasa@gmail.com

DOI: $10.14260 /$ jemds $/ 2019 / 98$

\section{(c) (1) $(9)$}

As medical laboratories have become to resemble high volume automated 'factories', they are producing millions of results, and the same standards of industrial manufacturing need to apply to the standards of medical laboratory testing. 3,4

The aim of this study is to evaluate the analytical performance of thyroid parameters in quality control of analytical phase of laboratory system by TS and sigma metric. Westgard rules were applied for the interpretation of daily internal quality control results. Westgard rules of $13 \mathrm{~s}, 22 \mathrm{~s}$, $\mathrm{R} 4 \mathrm{~s}, 41 \mathrm{~s}$ and $10 \mathrm{x}$ were considered as rejection and $12 \mathrm{~s}$ as warning situations for the respective run. Our laboratory is participating monthly in the external QC survey of RIQAS (Randox International quality assessment scheme, Randox Laboratories, United Kingdom) from which we retrieve the TS, SDI and \% Deviation results calculated from the peer group data.

\section{MATERIALS AND METHODS}

Our objective is to present the Target scoring (TS) and sigma metrics observed in our clinical chemistry laboratory in Gandhi hospital, Hyderabad, a tertiary care hospital in the Telangana state in India. The study period was from August to October 2018, i.e., for 3 months. Thyroid function testing i.e., T3 total, T4 total and Thyroid Stimulating Hormone (TSH); daily Quality Control data was taken from Siemens 
ADVIA Centaur XPT Immunoassay analyser for the period of 3 months. As we run only one level of Control material (BioRad) we had CV data for L1 only. QC material was assayed before commencing reporting of patient samples every day. We have validated quality control of our lab by calculating 3 months mean from the data of internal QC from the Advia Centaur XPT analyser data and online reports from the RIQAS
(External Quality Assurance Scheme) website to establish the coefficient of variation and bias respectively, for each analyte.

The TS relates the \% Deviation of the lab result from the Mean to a Target Deviation for Performance Assessment (TDPA). TDPAs are set to encourage participants to achieve and maintain acceptable performance. ${ }^{5}$

$\mathrm{TS}=\log _{10}(3.16 \mathrm{x}$ TPA $\div \%$ Dev $) 100$

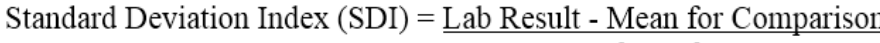 SDPA adjusted}

TDPAs are also used to calculate the Standard Deviation for Performance Assessment (SDPA). SDPA = Standard Deviation for Performance Assessment, calculated from TDPA and the Mean for Comparison.

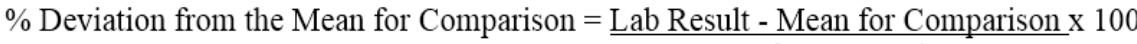

\section{Mean for Comparison}

\section{Total Allowable Error}

TEa is a model that combines both imprecision and bias (Trueness) of a method to calculate the impact on a test result. Analytical Quality Requirements are defined by Clinical Laboratory Improvement Amendment (CLIA) -88 Proficiency Testing Criteria in terms of total allowable error "TEa" (or more correctly "total allowable variation") for acceptable performance for each analyte. The most recent and extensive listing of biologic goals has been provided by Ricos et al., which is taken as reference value. These values are in accordance with CLIA guidelines. ${ }^{6,7}$

\section{Bias}

The difference between the average value and the true value is the bias, which is expressed numerically and so is inversely related to the trueness. Bias was taken from \% of Deviation of the peer group data from results returned from RIQAS; Bias $(\%)=$ (mean of all laboratories using same instrument and method -our mean) /mean of all laboratories using same instrument and method) X 100

\section{Coefficient of Variance}

The degree of precision is usually expressed on the basis of statistical measures of imprecision, $\mathrm{CV} \%$. CV was determined from the calculated laboratory mean and calculated standard deviation procured from the internal $\mathrm{QC}$ data over the 3 months:

$\mathrm{CV}(\%)=($ Standard deviation / Laboratory mean $) \mathrm{X} 100$ The sigma metrics for the various analytes was calculated by the following equation:

$\sum(\sigma)=($ TEa - bias $) / C V$

This form of the equation assumes all variables will be expressed in percentages, and the bias will be an absolute percentage (the presence of any bias always shrinks the allowable error, never enlarges it). ${ }^{8}$

All calculations were done in the spread sheet, MS excel of Windows 7.

\section{RESULTS}

The inaccuracy data for the TFT parameters that is $\%$ of Deviation (Bias) from the peer group data were obtained from RIQAS online reports. TS values from those reports and
SDI as well were shown in Table 1 . We have calculated (Average of daily CV) monthly CV \% (Imprecision) and bias (Inaccuracy) and calculated sigma metrics for each of 3 parameters for Level L1, shown in Table 2. Cumulative sigma metrics for the 3 months period for the control L1, shown in Table 3.

\begin{tabular}{|c|c|c|c|c|}
\hline SDI & AUG & SEP & OCT & Average \\
\hline T3 & -0.26 & 1.4 & -2.2 & 0.35 \\
\hline T4 & -0.73 & -0.58 & -0.08 & 0.46 \\
\hline TSH & -0.11 & -2.86 & -1.93 & 1.63 \\
\hline \multicolumn{5}{|c|}{ \% DEV } \\
\hline T3 & -2.4 & 12.8 & -21.2 & 3.60 \\
\hline T4 & -6.3 & -5 & -0.7 & 4.00 \\
\hline TSH & -0.8 & -21.6 & -14.4 & 12.27 \\
\hline \multicolumn{5}{|c|}{ Total Score } \\
\hline T3 & 120 & 57 & 35 & 71 \\
\hline T4 & 85 & 95 & 120 & 100 \\
\hline TSH & 120 & 26 & 43 & 63 \\
\hline Table 1. Month Wise SDI, \% DEV and TS Results From
\end{tabular}
RIQAS

\begin{tabular}{|c|c|c|c|c|c|}
\hline \multicolumn{6}{|c|}{ August } \\
\hline & TFT & TEa\% & BIAS\% & CV\% & sigma \\
\hline 1 & T3 & 9.22 & 2.4 & 10 & 0.7 \\
\hline 2 & $\mathrm{~T} 4$ & 7 & 6.3 & 13.8 & 0.1 \\
\hline 3 & TSH & 23.7 & 0.8 & 11.22 & 2.0 \\
\hline \multicolumn{6}{|c|}{ Sep } \\
\hline & TFT & TEa\% & BIAS\% & CV\% & sigma \\
\hline 1 & T3 & 9.22 & 12.8 & 10.1 & -0.4 \\
\hline 2 & T4 & 7 & 5.0 & 11.7 & 0.2 \\
\hline 3 & TSH & 23.7 & 21.6 & 11.2 & 0.2 \\
\hline \multicolumn{6}{|c|}{ Oct } \\
\hline & TFT & ТЕa\% & BIAS\% & CV\% & sigma \\
\hline 1 & T3 & 9.22 & 21.2 & 13.8 & -0.9 \\
\hline 2 & $\mathrm{~T} 4$ & 7.0 & 0.7 & 11.8 & 0.5 \\
\hline 3 & TSH & 23.7 & 14.4 & 11.2 & 0.8 \\
\hline \multicolumn{6}{|c|}{ Table 2. Monthly Bias, CV and Sigma Metrics for TFT } \\
\hline
\end{tabular}

\begin{tabular}{|c|c|c|c|}
\hline Average (3) Months & T3 & T4 & TSH \\
\hline CV (Siemens Machine) & 11.3 & 12.4 & 11.2 \\
\hline BIAS \% (RIQAS) & 12.1 & 4 & 12.3 \\
\hline Sigma & $\mathbf{0 . 3}$ & $\mathbf{0 . 2}$ & $\mathbf{1 . 0}$ \\
\hline Table 3. Cumulative Sigma Metrics \\
\hline
\end{tabular}




\begin{tabular}{|c|c|c|c|c|c|}
\hline 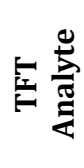 & $\underset{\tilde{z}}{\tilde{z}}$ & 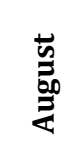 & के & 㝴 & 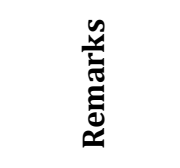 \\
\hline \multirow{3}{*}{$\begin{array}{c}\text { Total } \\
\text { T3 }\end{array}$} & TS $>50$ & 120 & 57 & $35^{*}$ & \multirow{3}{*}{$\begin{array}{c}\text { Sigma }<3 \\
\text { October } * \\
\text { Poor } \\
\text { Performance }\end{array}$} \\
\hline & $\mathrm{SDI}<2$ & -0.26 & 1.4 & $-2.2^{*}$ & \\
\hline & $\begin{array}{l}\% \text { Dev } \\
<15.10\end{array}$ & -2.4 & 12.8 & $-21.2^{*}$ & \\
\hline \multirow{3}{*}{$\begin{array}{c}\text { Total } \\
\text { T4 }\end{array}$} & TS & 85 & 95 & 120 & \multirow{3}{*}{$\begin{array}{c}\text { Sigma }<3 \\
\text { Good Overall } \\
\text { Performance }\end{array}$} \\
\hline & SDI & -0.73 & -0.58 & -0.08 & \\
\hline & $\begin{array}{r}\% \text { Dev } \\
<14.20\end{array}$ & -6.3 & -5 & -0.7 & \\
\hline \multirow{3}{*}{ TSH } & $\mathrm{TS}$ & 120 & $26^{*}$ & $43^{*}$ & \multirow{3}{*}{\begin{tabular}{|c} 
Sigma $<3$ \\
September* \\
Poor \\
Performance
\end{tabular}} \\
\hline & SDI & -0.11 & $-2.86^{*}$ & -1.93 & \\
\hline & $\begin{array}{l}\text { \% Dev } \\
<12.4\end{array}$ & -0.8 & $-21.6^{*}$ & -14.4 & \\
\hline \multicolumn{6}{|c|}{$\begin{array}{l}\text { Table 4. Total T3, Total T4 and TSH: Is The Parameter } \\
\text { Result Within Acceptable Limits Of Performance? }\end{array}$} \\
\hline
\end{tabular}

\section{DISCUSSION}

EQA is most concerned with detecting analytical errors i.e. errors that occur during the analysis of the sample. It may be possible that, after extensive investigations, the root cause of the poor performance cannot be established. Poor performance for a single sample could be attributed to random error. If poor performance has been noted for several samples, a systematic error is the most likely cause and the analytical process should be reviewed. The effectiveness or impact of any corrective actions taken can be assessed by continuing to monitor analytical performance over time. ${ }^{2}$

Our EQAS results for the three months showed two thirds of good performances for the three thyroid function testing parameters are summarized in Table 4. In August, the 3 tests were performing well but the sigma score was less than 3 . For the month of September, RIQAS results showed well performing T3 and T4 but TSH was having a very low TS; and SDI, \%Deviation were outside limits. In October, T3 results were not acceptable and TSH analysis needed improvement. The consistent analytical performance of T4 was good for all the three months of study. Our lab sigma metrics were below three sigmas. The imprecision of our lab is bigger which has led to a lower sigma in spite of reasonable bias values as shown in Tables 1, 2 and 3.

RIQAS reports of external quality assurance allows to compare the lab results with statistically robust consensus means and identify acceptable and poor performance using fit-for-purpose performance indicators: SDI, \%Deviation (Rapid assessment of concentration related biases) and Target Score (This chart provides a numerical index of performance, allowing at-a-glance assessment). Acceptable performance criteria: SDI < 2; Target score $>50$; the closer a value is to 120 , the better the performance, $<40$ is unacceptable; and \%Deviation should be less than the defined acceptable limits which is unique to each analyte, the closer the value is to zero, the better is the performance. TDPAs are fit-for-purpose performance criteria which are set taking guidance from ISO/IEC17043, ISO13528 and IUPAC. As those TDPAs take account of potential variability in samples through testing of homogeneity, reconstituted stability, real time stability and shipping/transport stability, this value is designed to be the upper deviation limit of performance for the each parameter. TDPAs are reviewed regularly and deemed fit for purpose by the RIQAS Advisory Panel.2,5 Hence our lab performances of testing analytes of thyroid are assumed to be under good and strict EQAS guidelines of RIQAS to achieve a acceptable quality as well as to introspect the $\mathrm{QC}$ strategies.

Our study presented the sigma metrics of ADVIA Centaur XPT Immunoassay analytical process results. Sigma metrics take into consideration of overall quality from the internal QC (imprecision, CV) and the External QC (Inaccuracy: Bias). Thus these results are helpful to apply QC strategies to minimize the analytical variance and get the optimal quality control procedures for improved overall quality assurance. As we have been taking the help of Westgard sigma rules, QC protocol needed to be customized for better outcome as well as consistent performance in Thyroid function tests on the Advia Immunoassay analyser.

For the analytes having less than three sigma value, Westgard sigma rules recommend a revision in the daily work load division, and to consider modification in QC procedure strategies. We are also required to adopt the changes in our protocol of number of shifts in a day. And a change in the frequency of daily runs is also required in our laboratory. For a more stringent QC practice, we also need to run 2 or 3 levels of daily controls covering range of the clinical decisive limits, for every analyte and their frequency must be increased.9-12 Iqbal $S$ et al in a study suggest that analytes required focused procedures (Choice of rejection rules and frequency of runs and quality control material measurements) for effective and improved quality control. ${ }^{13}$ The focused QC protocols will help to minimize the unnecessary QC monitoring hence reducing the cost for analytes with the high sigma metric result and to improve the $\mathrm{QC}$ of low sigma value analytes. However, false rejections rate should also be kept in mind which can be minimized by relaxing control limits up to $3 \mathrm{SD}$. And consider a possibility to implement a newer and better method because quality of the test may not be assured even after multiple QC cycles. ${ }^{14}$

Six sigma is the microscope of quality scientists. It shows the reality and does not mask problems. The errors that we are interested in are primarily analytical errors, which represent only the tip of the iceberg. However, until we see the whole iceberg and tackle, then it will be possible to reach Six Sigma level and even higher quality in clinical laboratories. ${ }^{15}$ When bias exists, it shifts our distribution away from the patient's true value. On the other hand, when we have a larger and larger imprecision, it spreads our distribution wider and wider. The combined impact of imprecision and bias may cause the thicker parts of the tails of our distribution to exceed the TEa, which means we are generating more defective test results. ${ }^{8}$

The clinical diagnostic laboratories are content if their results enclose \pm 2 SD or \pm 3 SD limits. In other words, they find defect rates of 45, 400 DPM opportunities and 2, 700 DPM opportunities as acceptable performance. Sigma value is inherently dependent on TEa definition given by various guidelines. We have different assigned values for a single analyte. Thus, if total allowable error of parameter is at higher side then there are more chances to get good sigma value, and to choose a TEa source is a choice of the laboratory. Unfortunately, these entire discrepancies mean right now is that there is neither standardization nor harmonization of the existing resources for TEa goals. ${ }^{8}$ In 
spite of getting acceptable CV some sigma values were not satisfactory. Upgraded analysers and better methodologies may help in achieving sigma values. ${ }^{16}$

EQA can allow seeing how a lab is performing across different concentrations and can be used in measuring the uncertainty of any bias. Six Sigma metrics for a laboratory can be calculated using IQC data if it incorporates all aspects of laboratory practice. These 2 factors (Target Score and Sigma metrics) are hence not comparable. ${ }^{5}$

The TS based published QC research (in detail) articles were very few from India till date and more discussion is needed for the RIQAS based quality tools.

\section{CONCLUSION}

The laboratory load of testing is ever increasing and analytical errors generation increases as well. With the advent of computerization and technological advancement in medical labs, the quality control of the performance of an analytical process is known by its precision and bias as compared to peer groups. By proper selection of TEa, QC strategy and the EQAS programme; and reducing the CV as far as possible and also keeping the bias to the minimum, any laboratory would achieve a perfect reporting of six sigma standard, which must be a continuous practice.

\section{REFERENCES}

[1] Carvalho GA, Perez CL, Ward LS. The clinical use of thyroid function tests. Arquivos Brasileiros Endocrinologia \& Metabologia 2013;57(3):193-204. https://dx.doi.org/10.1590/S0004-

27302013000300005.

[2] LT033-RIQAS-Explained-SEP 18: Randox catalogue. Accessed on 25 $5^{\text {th }}$ December 2018 http://pdf.medicalexpo.com/pdf/randox-

laboratories/lt033-riqasexplained/69798173358.html.

[3] Westgard S, Bayat H, Westgard JO. Analytical Sigma metrics: a review of Six Sigma implementation tools for medical laboratories. Biochemia Medica 2018;28(2):020502.

[4] Westgard JO. Six Sigma QC design and control: desirable precision and requisite $\mathrm{QC}$ for laboratory measurement processes. Madison (WI): Westgard QC 2001.

[5] Picton S. TDPA explain-regarding: Randox Laboratories/RIQAS to $<$ C. Author $>$ Personal communication. 8:37 PM, Dec 27th 2018.
[6] Farr AJ, Freeman KP. Quality control validation, application of Sigma metrics, and performance comparison between two biochemistry analysers in a commercial veterinary laboratory. J Vet Diagn Invest 2008;20(5):536-44.

[7] Ricos C, Alvarez V, Cava F, et al. Current databases on biological variation: pros, cons and progress. Scand J Clin Lab Invest 1999;59(7):491-500. This database was most recently updated in 2014

[8] Westgard JO, Westgard SA. The quality of laboratory testing today: an assessment of Sigma metrics for analytic quality using performance data from proficiency testing surveys and the CLIA criteria for acceptable performance. Am J Clin Pathol 2006;125(3):343-54. https://doi.org/10.1309/ V50H4FRVVWX12C79.

[9] Eggert AA, Westgard JO, Barry PL, et al. Implementation of a multirule, multistage quality control program in a clinical laboratory computer system. J Med Syst 1987;11(6):391-411.

[10] Lunetzky ES, Cembrowski GS. Performance characteristics of Bull's multirule algorithm for the quality control of multichannel hematology analysers. Am J Clin Pathol 1987;88(5):634-8.

[11] Menendez GR, Cabrera CO. Selection of an optimal combination of decision criteria for the internal quality control in an automatic analyser. Clin Chim Acta 2000;292(1-2):139-47.

[12] Coskun A. Westgard multirule for calculated laboratory tests. Clin Chem Lab Med 2006;44(10):1183-7.

[13] Iqbal S, Mustansar T. Application of Sigma metrics analysis for the assessment and modification of quality control program in the clinical chemistry laboratory of a tertiary care hospital. Indian J Clin Biochem 2017;32(1):106-9. https://doi. org/10.1007/s12291016-0565-x.

[14] Modi N, Gamit D. Quality assurance in thyroid profile with the six Sigma matrix. Int J Res Med 2017;6(4):325.

[15] Coskun A, Inal T, Unsal I, et al. Six Sigma as a quality management tool: evaluation of performance in laboratory medicine. Quality Management and Six Sigma 2010. ISBN: 978-953-307-130-5.

[16] Gami B, Patel D, Chauhan K, et al. Sigma metrics as a quality marker for analyzing electrolytes in laboratory. International Journal of Advanced Research 2013;1(7):197-201. 